\title{
Abstracts of papers presented at the Mammalian Genetics Group meeting, held in the Linnean Society Rooms, Piccadilly, London, on 25 and 26 November 1982
}

\section{Phenotypic expression of paternally and maternally derived $X$-chromosomes in liver lobes of female mice}

\author{
BY TH. BÜCHER, H. M. RABES AND J. T. NIELSON \\ Institutes of Physiological Chemistry (Th.B.) and of Pathology (H.M.R.), \\ University of Munich, Institute of Molecular Biology, \\ University of Aarhus (J.T.N.)
}

In mammalian females heterozygous for $X$-linked markers a natural system of mosaicism is available for developmental studies. The basic idea underlying such studies is that the determinative events of early development are random sampling events, so that statistical analyses of the compositions of differentiated mosaic tissues can yield information on the determinative events themselves (Nesbitt, Developmental Biology 26, 252-263, 1971). Several models have been elaborated (Kane, Genetic Mosaics and Chimeras in Mammals, 1978). Briefly the methodology involves measurements and analysis of variation in mosaic composition $(a)$ among mosaic individuals, $(b)$ of variation and correlation in composition among different tissues within these individuals, and (c) from sample to sample within the tissues. During studies of the clonal growth of carcinogen-induced preneoplastic cell populations in mouse liver striking correlations in mosaic composition between some but not all of six lobes of the liver were observed (Rabes et al., Cancer Research $42,3220-3227,1982$ ). At least two developmentally related groups of lobes could be discriminated. Phosphoglycerate kinase alloenzymes served as markers of hepatocytes expressing the maternally (PGK-1A) and the paternally (PGK-1B) derived $X$ chromosomes. Whereas the observed correlations of right and left liver lobes are of the same order as for tissues of diverse origin (e.g. medialis dexter and musculus gracilis of right hind leg, $r=0 \cdot 35$ ), and also comparable to the correlation of right and left lung reported by Nesbitt (1971), significantly closer correlations are found between right and right liver lobes and left and left liver lobes respectively. Our findings suggest an origin of mouse liver from at least two primordial centres. We thank Dr Victor E. Kane, Oak Ridge, for preliminary analyses of our data. 


\title{
Variation in genotype proportions in litters sired by chimaeric mice
}

\author{
BY MIA L. BUEHR \\ M.R.C. Mammalian Development Unit, Wolfson House, 4 Stephenson Way, \\ London NW1 2HE
}

Two male chimaeric mice of the constitution C57BI/6↔BALB/c were allowed to mate freely with BALB/c females. Two types of progeny were produced: albinos and agoutis representing fertilization of the BALB/c eggs by BALB/c and C57 sperm respectively. The proportion of each type, however, varied significantly from litter to litter. This variation showed no clear cyclical pattern and appeared unrelated to the age of the male or to the particular mother of the litter. Females were then put with the males overnight $(1800-0900)$ or during the day $(0900-1800)$ in mouse rooms with a dark period extending from 1900 to 0500 . Overnight matings resulted in a slight increase in the percentage of albino offspring, but day matings produced a highly significant increase in the proportion of agouti young. The proportions of progeny genotypes in litters of these chimaeric males are therefore thought to be affected by the time of mating relative to the time of ovulation in the female.

\section{PGK-1 expression in $P g k-1^{\mathrm{a}} / P g k-I^{\mathrm{b}}$ female mice associated with $X c e$ allele substitutions}

\author{
By B. M. CATTANACH, TH. BÜCHER AND S. J. ANDREWS \\ M.R.C. Radiobiology Unit, Harwell, Didcot, Oxon OX11 ORD, U.K. \\ Pathologisches Institut and Institut für Physiologische Chemie, \\ Universitat München, München 2, West Germany
}

An unequal $X$ chromosome expression due to non-random $X$-inactivation is found in female mice heterozygous for alleles at the $X c e$ locus. Thus, cells carrying the top allele $\left(X c e^{\mathrm{c}}\right)$ predominate over cells carrying the bottom allele $\left(X c e^{\mathrm{a}}\right)$ in $X c e^{\mathrm{a}} / \mathrm{X} c e^{\mathrm{c}}$ heterozygotes. This has been most clearly demonstrated in mice of genotype $P g k-1^{\mathrm{a}} X c e^{\mathrm{c}} / P g k-1^{\mathrm{b}} X c e^{\mathrm{a}}$ in which the proportion of PGK-1B allozyme is typically only about $30 \%$ of total allozyme. It would be expected that $X$ inactivation would be random in Xce homozygotes and the proportions of the two allozymes would be equal. Likewise, in mice of $P g k-1^{\mathrm{a}} X c e^{\mathrm{a}} / P g k-1^{\mathrm{b}} X c e^{\mathrm{c}}$ genotype $X c e$ allele heterozygosity should cause the proportion of PGK-1B allozyme to be about $70 \%$. Although recombinant $P g k-1^{\mathrm{a}} X c e^{\mathrm{a}}$ and $P g k-1^{\mathrm{b}} X c e^{\mathrm{c}}$ chromosomes have been obtained, first attempts to confirm the above expectations failed, perhaps due to the presence of other marker genes $\left(M o^{\text {blo }}, T a\right)$ in the crosses. These marker genes have now been removed by further recombination. The results of preliminary experiments using the new recombinant chromosomes now suggest that the proportion of the two PGK-1 allozymes in $X c e^{\mathrm{a}}$ and $X c e^{\mathrm{c}}$ homozygotes 
is near-equal, in accord with expectation. However, although the proportion of PGK-1B was found to be in excess of $70 \%$ in some $P g k-1^{\mathrm{a}} X c e^{\mathrm{a}} / P g k-1^{\mathrm{b}} X c e^{\mathrm{c}}$ heterozygotes the mean score of animals of this genotype was only a little above $50 \%$. This result could be a valid one or an artifact of small numbers. Further data are being collected.

\title{
Studies on steroid sulphatase in mice
}

\author{
BY I. CRAIG AND A. J. M. CROCKER \\ Genetics Laboratory, Department of Biochemistry, University of Oxford, \\ South Parks Road, Oxford OX13QU
}

Both human and woodlemming steroid sulphatase (STS) levels have previously been found to be proportional to the number of $X$ chromosomes present, indicating that the Sts locus is an unusual $X$-linked gene in that it is not subject to $X$-inactivation. In this study STS levels were investigated in $X X$ female and $X Y$ male fibroblasts from mice, as well as in $X X$ female, $X X$ (sex reversed) male and $X Y$ male liver extracts. The STS levels were similar in both $X X$ and $X Y$ fibroblasts and in the liver extracts. It is concluded that, although the evidence is perfectly compatible with $X$-linkage of Sts in the mouse, if $S t s$ is $X$-linked the locus is subject to $X$-inactivation.

\section{Sxr, a cytological re-visit}

\author{
By E. P. EVANS,* M. D. BURTENSHAW* and B. M. CATTANACH $†$ \\ * Sir William Dunn School of Pathology, University of Oxford, \\ South Parks Road, Oxford OX1 3RE \\ † M.R.C. Radiobiology Unit, Harwell, Didcot, Oxon OX11 ORD
}

Since the sex-reversed $(S x r)$ factor in the mouse was first described there have been a number of unsuccessful attempts to relate its presence to some cytologically visible chromosome event at either meiosis or mitosis. Because the factor showed an apparent autosomal inheritance and caused chromosomal females to develop as phenotypic males, most attention was concentrated on the autosomes and the $Y$ chromosome. Earlier this year, however, the in situ hybridization studies of Singh \& Jones (1982) re-focused attention away from the autosomes and on to the two sex chromosomes. We re-focused with G-banding and in bone marrow cells showed the presence of a dark-staining distal body on the $Y$ chromosome in $X / Y S x r$ males and on one $X$ chromosome in $X / X S x r$ males. Studies at diakinesis in the former showed that the transfer of this Sxr-related chromatin was accomplished by crossing over from one chromatid of the $Y$ on to that of an $X$ to produce $X, X S x r, Y$ and $Y S x r$ sperm in equal numbers. This represents the first visible evidence of crossing over between $X$ and $Y$ in the mouse, and its implications in relation to male mice not carrying $S x r$ will be discussed. 


\title{
$X Y$ sex reversal in mice
}

\author{
By A. GROPP AND H. WINKING \\ Institut für Pathologie Medizinische Hochschule, Lübeck
}

A consomic strain with the wild mouse-derived $\mathrm{Rb}(4.12) 9 \mathrm{Bnr}$ metacentric and the appertaining $Y$ chromosome on $\mathrm{C} 57 \mathrm{BL} / 6 \mathrm{~J}$ shows a highly distorted sex ratio, with an excess of females and impaired fertility of a considerable part of the male and female progeny. On serial karyotyping of animals from subsequent litters $60 \%$ females with $X X$ chromosomes were observed, $20 \%$ were phenotypic females with an $X Y$ karyotype, and $20 \%$ were males with $X Y$ chromosomes. Dissection of the $X Y$ females revealed in most cases an entirely female genital tract with small ovaries and few follicles. Others were lateral hermaphrodites with dystrophic tubules in the testis part. Fertility, though with small testes, occurred in some of the $X Y$ males, which guarantee the maintenance of the strain. Other males were sterile, showing impaired spermatogenesis or true hermaphroditism, sometimes with active spermatogenesis in the testis part and endocrine stimulation of the interstitial cells in the ovary part. In essence these findings confirm the observations reported by E. Eicher et al. (Science, 217, 535-537, 1982).

"The mechanism of $X Y$ sex reversal is dependent on and transmitted with the $Y$ chromosome of feral origin. Since the $Y$ chromosome is derived from males of the subspecies $M$. m. domesticus, the genetic mutation on different $Y$ chromosomes has to be defined by further studies in terms of intraspecific diversity and distance. However, the sex reversal phenomenon is also related with the properties of the genetically highly specialized $\mathrm{C} 57 \mathrm{BL} / 6 \mathrm{~J}$ strain, and the assumption is made that it is possible to induce similar phenomena with a diversity of changes transferred onto the genome of this strain. The established consomic strain C57BL/6-Rb9Bnr $Y^{g r}$ might be a useful tool for further systematic studies.

\section{Genetics of lidgap in mice}

\author{
BY MURIEL J. HARRIS AND DIANA M. JURILOFF \\ University of British Columbia, Department of Medical Genetics, \\ Vancouver, B.C., Canada, V6T 1W5
}

Lidgap, a defect in eyelid development in mice expressed as open eyes at birth, is an example of a genetically complex morphological defect in mammals. Many separately occurring spontaneous mutations result in this phenotype. Allelism tests indicate that four mutations are at different loci $(l g, g p, o e, o e l)$ and that four are likely at one of these loci, called lidgap $\left(l g, l g^{s t n}, l g^{M l}, o a\right)$. Only oe has been mapped. All have been assumed to be single autosomal recessive mutations, but outcrosses show that the genetic pattern is often more complex. We have tested the hypothesis that a single lidgap gene can be sufficient to cause the trait. It can. 
We are now beginning to unravel the more complex situations, with evidence that: (1) whereas $l g$ is sufficient to cause lidgap, $l g^{s t n}$ requires a helper locus from the mutant stock; (2) expression of $l g$ can be masked by a recessive allele at an unlinked suppressor locus, this allele being present in a normal ICR stock. Also, a pre-natal injection of cortisone on day 14 cures lidgap in most $\left(l g^{M l}\right)$, some $\left(l g^{s t n}\right)$, or few $(\mathrm{lg})$ foetuses. The consistent rank order of the three lidgap alleles in frequency of affected offspring in allelism, outcross and cortisone tests suggests to us the conceptual framework of progressively more defective hypomorphs at the lidgap locus $l g^{M l}<l g^{g t n}<l g$, with $o a$ being a strong, but perhaps only partially overlapping, allele.

\title{
Genetic variation in antibody responses to schistosome infection in mice
}

\author{
BY JANET T. JONES \\ Department of Biochemistry, University of Glasgow, Glasgow G12 8QQ
}

Strains of mice vary in their development of resistance to reinfection with Schistosoma mansoni both during chronic infection (Bickle et al., Experimental Parasitology 50, 222-232, 1980) and following immunization with irradiated cercariae (Murrell et al., J. Parasitology 65, 829-831, 1979). Studies of mouse strains which vary in their antibody response to infection may help to define the role of antibody in both resistance to re-infection and in the pathology of the disease. The antibody response of several inbred strains of mice to adult worm antigen and to egg antigen has been measured using an ELISA assay. There are genetically controlled differences in the magnitude and timing of the antibody response to these complex antigen mixtures. Antibody responses have also been studied in four congenic strains of mice, and it has been found that both the $\mathrm{H}-2$ type of the mouse and its genetic background had an effect on the antibody titre against worm antigens. Co-precipitation of radio-iodinated worm antigens with sera from infected mice has been used to identify the major antigens involved in the antibody response, and to try to detect genetic variation in responses to particular antigens.

\section{X-ray-sensitive mutants of Chinese hamster ovary cells}

\author{
BY L. M. KEMP, P. A. JEGGO AND R. HOLLIDAY \\ Genetics Division, National Institute for Medical Research, The Ridgeway, \\ Mill Hill, London NW7 1 AA
}

The microbial 'toothpick' technique, whereby cells from isolated colonies can be transferred to gridded replica agar plates, has been adapted for use with Chinese hamster ovary (CHO) cells. Using this method, several X-ray-sensitive mutants of this cell line have been isolated. Their $\mathrm{X}$-ray sensitivity covers a wide range of 
values, with the most sensitive having a $D_{0}$ value of $15 \mathrm{rad}$, compared to a $D_{0}$ value of $100 \mathrm{rad}$ for wild-type. Preliminary characterization of these mutants is in progress, including cross-sensitivity to other DNA damaging agents, repair of single-strand breaks, unscheduled DNA synthesis and chromosome damage. So far, some of these mutants have been shown to be sensitive to other DNA-damaging agents, including UV, ethyl methane sulphonate (EMS) and methyl methane sulphonate (MMS).

\title{
Is DNA methylation correlated with human $X$-chromosome inactivation?
}

\section{BY SUSAN LINDSAY AND MARILYN MONK \\ M.R.C. Mammalian Development Unit, 4 Stephenson Way, London NW1 2HE}

We examined methylation at CCGG sites in active and inactive human $X$ chromosomes, using MspI and HpaII digestion of genomic DNA from human fibroblast or lymphoid cells (provided by L. Huschtscha) of $X^{+} X^{-}, X^{+} O, X^{+} Y$ and $X^{+} X^{-} X^{-} X^{-} Y$ karotype. The digested DNA was run on agarose gels, transferred to filters, and hybridized to four different cloned Eco R1 fragments specific to the human $X$ chromosome (probes provided by $\mathrm{K}$. Davies and R. Williamson). All four probes show variable methylation of the CCGG sites in their vicinity, indicating a cell-to-cell variation within a particular culture. The patterns of methylation observed are the same irrespective of the numbers of active and inactive $X$ chromosomes and of the origin of the cell lines.

\section{An investigation of a genetic contamination in two strains of mice imported from the USA}

\author{
By D. P. LOVELL and P. TOTMAN \\ M.R.C. Laboratory Animals Centre, Woodmansterne Road, Carshalton, \\ Surrey SM5 $4 E F$
}

Genetic contaminations are unfortunately proving to be a common occurrence in the colonies maintained by commercial breeders and academic institutes in both the United Kingdom and the U.S.A. (Festing \& Lovell, 7th ICLAS Symposium, 1980; Kahan et al., Science 217, 379-381, 1982). In 1981 the LAC imported two congenic strains of mice from the U.S.A. for a research worker in the U.K. and subsequently re-derived them. In the course of development of electrophoretic methods for genetic monitoring it was discovered that these two strains were segregating for two out of three genetic loci investigated. A subsequent reimportation this summer showed a similar pattern implying that the colonies had been contaminated for over 18 months. These animals have been used to investigate and compare a series of methods of genetic monitoring: mandible analysis, coat colour markers and electrophoretic markers. Results of these studies will be presented here. 


\title{
Cell selection in embryos heterozygous for Searle's $X$-autosome translocation
}

\author{
BY ANDY McMAHON AND MARILYN MONK \\ M.R.C. Mammalian Development Unit, Wolfson House, 4 Stephenson Way, \\ London NW1 $2 H E$
}

To investigate whether the preferential expression of genes on the translocated $X$ chromosome in female mice carrying the $X$-autosome translocation $\mathrm{T}(X ; 16) 16 \mathrm{H}$ (Searle's translocation) is due to non-random inactivation or to cell selection, we examined tissues of mouse embryos heterozygous for the $X$-linked gene coding for phosphoglycerate kinase $(P g k-1)$. From the cross T16H Pgk-1 ${ }^{b} /+P g k-1^{a} \nmid \times$ $+P g k-1^{a} / Y_{0}$, embryos expressing both isozymic forms of PGK-1 in the epiblast, and only the maternally inherited $P g k-1^{b}$ allele in extra-embryonic tissues, were assumed to be chromosomally balanced, heterozygous female embyos carrying the Searle's translocation (like the mother). The normal $X$ chromosome in this cross carries a high-expression $X c e^{c}$ locus. At 6 days post-coitum (p.c.) both isozymes were equally expressed in the epiblast as expected if both $X$ chromosomes are active, but by 7 days p.c., the PGK-1B contribution was significantly less than $50 \%$, suggesting that $X$ inactivation has occurred with a bias towards inactivation of the translocated $X$ chromosomes carrying the lower-expression Xce allele. By 8 days p.c. the situation was the reverse with a $P g k-1^{b}$ contribution of significantly more than $50 \%$, and by $12 \frac{1}{2}$ days p.c., no $P g k-1^{a}$ expression could be detected. We interpret the dramatic change in isozyme expression between 7 and 8 days p.c. as indicating rapid selection against cells that had inactivated the translocated $16^{X}$ chromosome.

\section{Pachytene chromosomes in male and female mice heterozygous for the Is $(7 ; 1) 40 \mathrm{H}$ insertion}

\author{
By SHANTHA MAHADEVAIAH AND URSULA MITTWOCH \\ Department of Genetics and Biometry, University College London, Wolfson House, \\ 4 Stephenson Way, London NW1 2HE
}

The Is40H insertion causes sterility in males with severe spermatogenic arrest. whereas female carriers are capable of reproduction. We have studied pachytene chromosomes in whole-mount preparations stained with silver nitrate in the light microscope. The insertion configuration appeared either as two bivalents or as a quadrivalent. The proportion of quadrivalents seen was lower in oocytes than in spermatocytes. Out of 36 oocytes, 16 cells contained 20 bivalents each, and in 5 of these all bivalents seemed to be completely synapsed. Out of 43 spermatocytes, 9 cells contained 20 bivalents and none of these showed complete synapsis. In all but 4 of the spermatocytes, an insertion configuration was either associated with the $X Y$ bivalent or adjacent to it. We plan to obtain more detailed information regarding the rearranged chromosomes by electron microscopy. 


\title{
Imprinting of the mouse $X$-chromosome and the Xce locus
}

\author{
By S. RASTAN AND B. M. CATTANACH \\ Divison of Comparative Medicine, Clinical Research Centre, Harrow \\ M.R.C. Radiobiology Unit, Harwell
}

In eutherian mammals, although either $X$ chromosome can be inactivated at random in somatic cells, the paternally derived $X$ chromosome is preferentially inactivated in certain extra-embryonic tissues. In yolk sac, the nonrandom element is confined to the endoderm layer in which there is primary non-random inactivation of the paternal $X$ chromosome. This non-random $X$-inactivation is caused by the paternal $X$ chromosome being 'imprinted', i.e. somehow marked as different, either during gametogenesis or fertilization. Centrally in the mouse $X$ chromosome is a locus, the $X$ chromosome-controlling element (Xce) locus which causes non-random $X$ chromosome activity. Three alleles of the $X c e$ locus are known: $X c e^{a}, X c e^{b}$ and $X c e^{c}$, which affect the probability of $X$-inactivation such that an $X$ chromosome carrying an $X c e^{a}$ allele is more likely to be inactivated than an $X$ chromosome carrying an $X c e^{b}$ allele in the same cell. The work described here was designed to elucidate whether the non-random inactivation of the imprinted paternal $\boldsymbol{X}$ chromosome in yolk sac endoderm can be modified, or even overridden, by the effect of different $X c e$ alleles. Embryos heterozygous for a marker $X$ chromosome (Cattanach's translocation) carrying different $X c e$ alleles on the paternally and maternally derived $X$ chromosomes were produced, their yolk sacs separated into their component endoderm and mesoderm layers and metaphase chromosomes prepared by the modified Kanda method. Individual cells at metaphase were then scored for whether the $X^{P}$ or the $X^{M}$ was dark-staining and inactive. The results show that the $X c e^{c}$ allele when present on the paternally derived $X$ can significantly reduce the proportion of inactive $X^{P}$ seen in yolk sac endoderm compared with controls.

\section{Sex reversal and $H-Y$ typing}

\section{By ELIZABETH SIMPSON, EVA EICHER AND PHILLIP CHANDLER}

Transplantation Biology Section, Clinical Research Centre, Watford Road, Harrow, Middlesex, HA1 $3 \mathrm{UJ}$

The Jackson Laboratory, Bar Harbor, Maine 04609, U.S.A.

It is generally true that whereas mammals having the sex chromosome constitution $X X$ are females, those with $X Y$ are males. The interpretation has been that a gene or genes on the $Y$ chromosome controls primary sex determination so that the bipotential gonad in the foetus becomes a testis when a $Y$ chromosome is present, and an ovary in its absence. Although the manner in which this occurs is incompletely understood, much circumstantial evidence is in accord with a current hypothesis that a male specific antigen, $H-Y$, coded or controlled by 
gene(s) on the $Y$ chromosome is the differentiation antigen that triggers testis formation. There are a number of exceptions to the rule that the presence of the $Y$ chromosome determines testis development in mammals. We have investigated the $H-Y$ typing of mice showing several different types of sex reversal. From the results, it appears that the testis determining gene $T d y$, which is normally located on the $Y$ chromosome, is not the sole determinant of testis development, but needs to interact with at least two autosome genes.

\title{
Pigment gene expression with particular reference to sash $\left(W^{\text {sh }}\right)$ chimaeras
}

\author{
BY D. A. STEPHENSON \\ M.R.C. Radiobiology Unit, Harwell, Didcot, Oxon OX11 ORD, U.K.
}

Several methods, using graft techniques, have been employed to investigate the influence of the local genetic environment of follicular melanocyte pigment production. As melanocytes and cells of the hair follicle are clonally derived from different numbers of independent embryological precursors the construction of chimaeras offers an alternative method. Chimaeras were constructed from either $W^{s h} W^{s h}$ or compounds of $W^{s h}$ with viable dominant spotting $\left(W^{v}\right)$ and beige fuzzy leaden homozygotes (bgbg $f z l n / f z l n)$. Both $W^{s h} W^{s h}$ and $W^{s h} W^{v}$ are black-eyed white mice. Lack of coat pigmentation is presumably due to the absence of follicular melanocytes. The $W$ locus is reported to act within the melanoblast affecting their migration from the neural crest. Thus, the melanocytes should arise, almost exclusively, from the $b g b g f z l n / f z l n$ cell pool. Fuzzy is expressed through the cells of the epidermis and provides an epidermal marker of follicular genotype. Analysis of non- $f z$ hairs would therefore indicate whether the genotype of the follicular environment modifies expression of the $b g$ and $l n$ loci. The distribution pattern of pigmentation produced by both types of chimaera (i.e. $W^{8 h} W^{s h} \leftrightarrow b g b g$ $f z \ln / f z \ln$ and $W^{s h} W^{v} \leftrightarrow b g b g f z \ln / f z l n$ ) was reminiscent of the heterozygous $W^{s h}$ phenotype. The pigment phenotype was similar to that produced by the $b g$ ln melanocytes. Bands of $f z$ and non- $f z$ hairs, when present, were distributed at random throughout the coat in both pigmented and non-pigmented regions. The significance of these observations will be discussed.

\section{A tiger-coloured mouse: an activated transposable element?}

\author{
By MARGARET E. WALLACE AND H. R. NASH \\ Department of Genetics, University of Cambridge, U.K. \\ Department of Zoology, University College, London, U.K.
}

After 6 sibmatings in a laboratory-bred colony of pure wild Peruvian house mice, a male appeared with an excessive rate of non-pairing of the $X$ and $Y$ chromosomes (Wallace, M.N.L. 63, 1980). The population is unique in giving an excessive 
number of mutants (Wallace, M.N.L. 61, 1979) and chromosomal damage (Wallace, M.N.L. 67, 1982). After the male was crossed with a laboratory stock, a tiger-coloured mouse, of $\frac{1}{8}$ Peru genome, appeared from a female $A^{y} A \times$ male $a^{t} a^{t}$. ( $A^{y}=$ yellow, $A=$ wild type, $a^{t}=$ black-and-tan, chromosome 2). This colour, and his production of an excess of black-and-tan over yellow progeny, indicates that he was a mosaic of $A^{y} a^{t}$ and $a^{t} a^{t}$ cells. 'Tiger's' genotype and progeny scores were normal for 12 allozyme loci. He was typed as $c c$ for $E s-3$, and his parents as $b c \times c c$. Progeny scores showed that he gave a big excess of $a$ over $c$ gametes when young, and only $c$ gametes when old. It seems he was a mosaic of $a a$ and $c c$ cells. These might have arisen by somatic crossing-over from an ac zygote in Tiger, and this in turn from a mutation from $b$ or $c$ to $a$ in his dam's ovum. There were four classes of progeny from the joint segregation at the $A^{y}$ and $E s-3$ loci. Two or more rare events in development, involving $A^{y}$, are needed to explain this. A new dominant, umbrous-patterned, $U p$, arose in a close relative. It gives a symmetrical pattern of large dark patches in $A^{y}$ genotypes, a different pattern for each $U p^{+}$ mouse. There were seven other unusual mice in 'Tiger's' close relatives, some probably somatic mosaics. 'Tiger's' yellow dam, $\frac{1}{4}$ Peru genome, was the progenitor of all the unusual mice. It is speculated that the ectropic murine leukaemia virus associated with yellow (Copeland et al. 1983) reacted with the chromosomedamaging agent in the Peru genome, and was transposed successively to different DNA sequences in 'Tiger' and his relatives.

\section{Genetics of halothane sensitivity in pigs}

\section{By A. J. WEBB ANd A. E. CARDEN \\ A.R.C. Animal Breeding Research Organisation, West Mains Road, Edinburgh EH9 $3 J Q$}

Pigs with an increased liability to porcine stress syndrome (PSS), involving sudden deaths and pale, soft, exudative (PSE) meat develop muscular rigidity during a 3-minute exposure to the anaesthetic halothane at around 8 weeks of age. The reaction is similar to malignant hyperpyrexia of humans. In pigs, halothane sensitivity is controlled by a single autosomal locus situated within a group of 4 blood-type loci in linkage disequilibrium. Compared with normal contemporaries, positive reactors show economically important advantages in lean content together with disadvantages in reproduction and PSS. The gene appears largely recessive for halothane sensitivity but additive for lean content, giving the heterozygote an economic advantage. Repeat tests on the same pigs show that the penetrance of the halothane-positive genotype is greatly reduced before 5 weeks of age. Gene frequency varies from 0 to $95 \%$ in world breeds, and can be rapidly changed by halothane screening or blood typing. For practical pig breeding, the challenge is now to devise methods of exploiting the advantage in lean content without the associated disadvantages of PSS and poor reproduction. 